\title{
DYHEARTMON - MONITORAMENTO AMBULATORIAL E RESIDENCIAL REMOTO DA PRESSÃO ARTERIAL BASEADO EM INTERNET DAS COISAS
}

\author{
Marcus Alexandre G. dos Santos, Victor Hugo C. de Albuquerque \\ Programa de Pós Graduação em Informática Aplicada - Laboratório de Bioinformática \\ - Universidade de Fortaleza (UNIFOR) - Fortaleza - CE - Brasil \\ marcus.santos@edu.unifor.br, victor.albuquerque@unifor.br
}

\begin{abstract}
Lifestyle is the main factor for the development of cardiovascular diseases. Therefore, proper health care can greatly reduce the likelihood of heart problems, as well as routine examinations and follow-up by a cardiologist. There are several conventional tests to support the diagnosis of cardiovascular diseases, which are often tiresome and costly to the patient. DyHEARTMon aims to be an alternative and complementary internet based tool of things for patients and health professionals associated with cardiovascular disease. Through a hybrid solution (smartband, smartphone and dashboard), the heart rate and blood pressure will be captured so that the potential risk of hypertension of the monitored individual is analyzed and presented, thus presenting an alternative to the Ambulatory Blood Pressure Monitoring (ABPM) and Home Blood Pressure Monitoring (HBPM).
\end{abstract}

Resumo. O estilo de vida é o principal fator para o desenvolvimento de doenças cardiovasculares. Portanto, os devidos cuidados com a saúde podem reduzir bastante as probabilidades de problemas cardíacos, assim como exames de rotina e acompanhamento por um cardiologista. Existem diversos exames convencionais para o apoio ao diagnóstico de doenças cardiovasculares, sendo estes muitas vezes cansativos e onerosos ao paciente. $O$ DyHEARTMon, tem como objetivo ser uma ferramenta alternativa $e$ complementar baseada em internet das coisas para pacientes e profissionais de saúde associados as doenças cardiovasculares. Através de uma solução hibrida (smartband, smartphone e dashboard), será captado a frequência cardíaca e a pressão arterial para que posteriormente seja analisado $e$ apresentado o potencial risco de hipertensão do indivíduo monitorado, apresentando desta forma uma alternativa para os exames de monitorização ambulatorial da pressão arterial (MAPA) e monitorização residencial da pressão arterial (MRPA).

\section{Introdução}

As doenças cardiovasculares estão associadas a distúrbios do coração e dos vasos sanguíneos, podendo causar arritmia cardíaca, acidente vascular cerebral, hipertensão, infarto e insuficiência cardíaca, sendo classificadas como doenças crônicas não transmissíveis, assim como o câncer, o diabetes e as doenças respiratórias crônicas, por exemplo. Diante da sua elevada prevalência e baixas taxas de controle, a hipertensão é reconhecida como um grave problema de saúde no país. Isto se deve à grande parcela 
de indivíduos hipertensos não diagnosticados ou não tratados adequadamente [VIGITEL, 2018]. Em face da situação, reforça-se a importância da verificação da pressão arterial como uma prática a ser adotada pelos profissionais de saúde em toda avaliação clínica.

O corpo humano pode dar sinais que não está funcionando em padrões que no futuro podem gerar agravos maiores. A inserção de múltiplos fatores de risco no desenvolvimento das doenças cardiovasculares implicam diretamente na gênese, progressão e ocorrência dos eventos cardiovasculares futuros [DAVIGNON e GANZ, 2004] e que enumerar os marcadores de risco para a doença cardiovascular tem um potencial significativo para melhorar a seleção dos indivíduos para estratégias preventivas [BERWANGER, 2005]. O risco cardiovascular global é definido como a probabilidade de um indivíduo ter um evento, por exemplo infarto do miocárdio, durante um período de tempo. Embora existam diferentes escores de risco cardiovascular, destacando-se o Escore de risco de Framingham para Doença Cardiovascular Geral e o projeto SCORE, a maioria deles considera informações como sexo, valor da pressão arterial, presença de tabagismo ou diabetes e perfil lipídico do paciente mediante coleta dos exames de colesterol total e colesterol HDL.

A adoção da internet das coisas (IoT) nos cuidados do coração, traz uma gama de novas oportunidades na maneira de como é abordado o diagnóstico e o tratamento de doenças cardíacas. Se antes o médico tinha de esperar o paciente ir até ele para que fosse realizada a anamnese inicial, hoje podemos através de uma rede de monitoramento em tempo real, informar o status atual do paciente, como também prever situações extremas como um ataque cardíaco que pode em muitos casos levar a morte ou deixar sequelas que são onerosas tanto para a estrutura de tratamento da saúde e principalmente para o próprio paciente.

Com base nos estudos e levantamentos realizados junto aos profissionais da saúde, identificamos que o monitoramento de sinais vitais associados a marcadores de risco para a hipertensão podem estimular um melhor controle da saúde por parte do paciente e demais envolvidos no cuidado do mesmo. Selecionamos o monitoramento dinâmico da pressão arterial sistólica associado a preditores como idade, sexo, peso, hábitos potencialmente prejudiciais a saúde e histórico familiar que serão coletados, monitorados e posteriormente apresentados através de dispositivos IoT. O DyHEARTMon - Monitoramento Dinâmico do Coração, tem como objetivo ser uma ferramenta computacional baseada em IoT de apoio para o paciente, cuidadores e profissionais de saúde associados a doenças cardiovasculares.

\section{Arquitetura do DyHEARTMon}

A proposta de realizar o monitoramento dinâmico do coração dando ênfase ao controle do risco em ter um episódio de hipertensão oferecendo uma alternativa de gestão com foco no indivíduo, utiliza uma infraestrutura dentro de uma Rede de Área Pessoal associada a uma Rede de Área Corporal sendo gerenciada na nuvem.

Os seguintes tópicos representados na Figura 1, foram avaliados para 0 desenvolvimento da proposta: 1) Mensuração de sinais - Identificar os bio sinais que devem ser mensurados. A captação da pressão sanguínea para um experimento relacionado a hipertensão, pois a aferição do mesmo será primordial para o monitoramento e posteriormente avaliar o nível de criticidade do estado físico do voluntário; 2) Método para captura de sinais - Selecionar o método que será utilizado 
para capturar os bio sinais pontuados na proposta do experimento; 3) Faixa do experimento - Definir a área de abrangência do experimento para que seja avaliado os recursos computacionais e humanos necessários para o sucesso da proposta; 4) Plataforma embarcada - Logo após definir a área de abrangência do experimento é necessário identificar e posteriormente avaliar os recursos computacionais que serão utilizados, inclusive pontuando o valor necessário que poderá ser investido em todo o projeto. Os recursos embarcados necessários para a proposta, tais como sensor para coleta de sinal corporal e módulo de comunicação para curta distância estão inseridos no smartband. Este smartband conta com recursos como monitoramento cardíaco e análise de sono, sendo possível checar a frequência cardíaca e pressão arterial mantendo um histórico no aplicativo móvel. O desenvolvimento procedural foi aplicado com o intuito de realizar pequenas tarefas no sistema, tais como agendamento da execução de importação, atualização das aplicações, desligamento automático e apresentação dos valores; 5) Plataforma de aplicação - A fim de alcançar os objetivos desta pesquisa, percebeu-se a necessidade de um ambiente de desenvolvimento para cálculos estatísticos e geração de gráficos e, ao mesmo tempo, para apresentar de maneira dinâmica os resultados das análises realizadas. Diante disso, o RStudio com o pacote Shiny integrado, apresentou todas as características necessárias a partir de requisitos levantados para a análise e apresentação de resultados; 6) Coletar amostras - A etapa final antes de colocar para o público um produto na área da saúde foi testá-lo em voluntários e buscar avaliação da proposta por parte de médicos com especialidade em cardiologia.

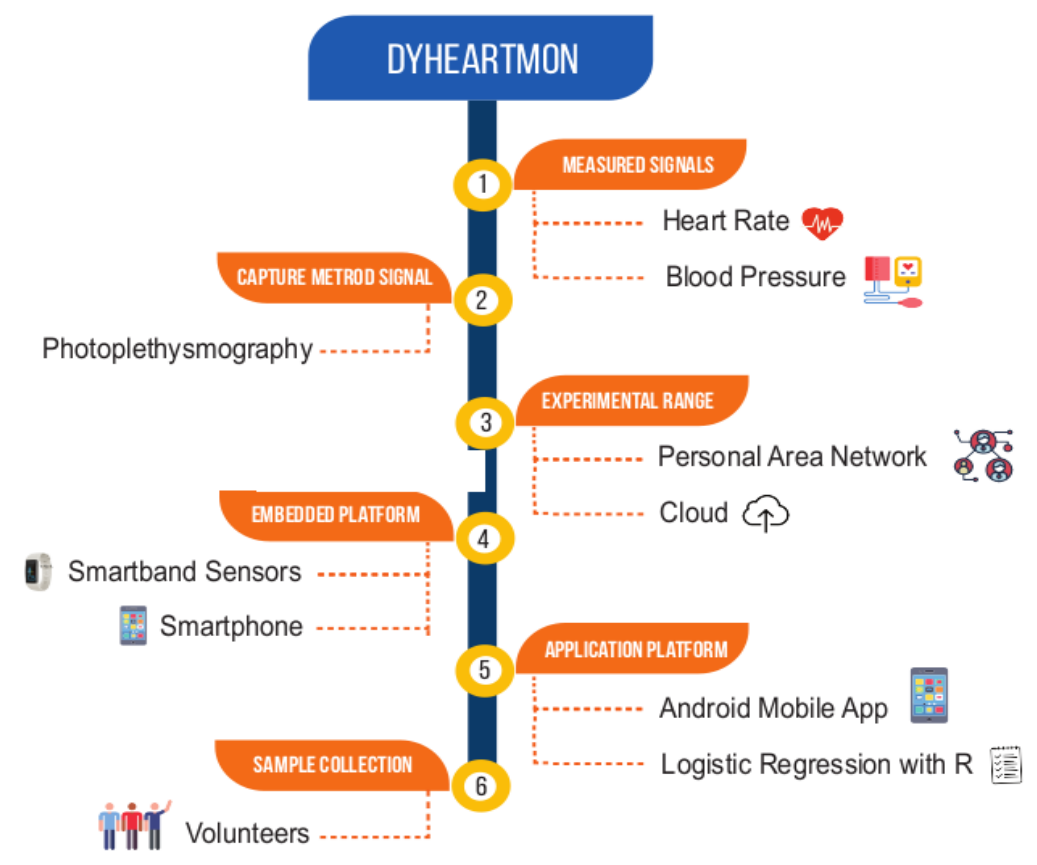

Figura 1. Tópicos avaliados para o desenvolvimento da proposta

A implementação da aplicação para a proposta alternativa do monitoramento e análise da pressão arterial tem como base o uso das medições coletadas no smartband Zucoor, para isso foi utilizado os dados armazenados no arquivo "change.log". Para a análise dos dados armazenados no arquivo "change.log", o mesmo é importado para o ambiente de desenvolvimento RStudio Desktop. As funções básicas de estatística do R 
já vêm implementadas. No entanto, funções mais avançadas requerem o uso de pacotes. Para a análise dos dados contidos no arquivo "change.log”, são necessários os pacotes: "bupaR”, “edeaR” e "ggplot2” e já Para a análise da relação de um efeito significativo da idade, colesterol total, frequência cardíaca, tabagismo, pressão arterial sistólica, pressão arterial diastólica na predição do diagnóstico de hipertensão, são necessários os pacotes: "LocalControl”, "riskRegression", "survival" e "SurvRegCensCov". O projeto DyHEARTMon para atender as necessidades especificas surgidas mediante suas características pontuais utilizou o conceito de script.

Os dashboards apresentam métricas que precisam ser acompanhadas para o bom desempenho da operação. As métricas é que ajudam os analistas a corrigir os erros e falhas nos processos aparecerão nos indicadores. Para a apresentação e acompanhamento dinâmico do monitoramento realizado a partir da solução proposta, foi desenvolvido um dashboard operacional. O dashboard consente o acompanhamento de indicadores que permitem aos médicos tomar decisões de curto e médio prazo com relação a saúde do paciente. Neste dashboard é possível acompanhar o resultado das coletas realizadas através do smartband, além de descobrir informações importantes como por exemplo, dentro do período monitorado, saber se o voluntário teve episódios pontuais ou constantes relacionados a oscilação da pressão arterial e/ou frequência cardíaca.

\section{Experimentos e Resultados}

Para avaliar a eficiência da proposta, foi realizado um comparativo com o exame tradicional de monitorização ambulatorial da pressão arterial (MAPA), utilizando tanto os recursos tradicionais de captação da pressão arterial e frequência cardíaca como o smartband durante o mesmo período de captação das amostras. Neste processo, o teste foi realizado utilizando ao mesmo tempo no braço esquerdo o manguito com a mangueira e no pulso o smartband no teste de esteira e posteriormente em paralelo no teste convencional MAPA com o smartband no pulso, com uma bolsa lateral com o aparelho convencional e o manguito. O smartband foi utilizado durante 72 horas, sendo que 24 horas antes do inicio da realização do exame convencional, 24 horas durante a realização do exame e 24 horas após a retirada dos recursos convencionais utilizados para a realização das medições necessárias para a geração do laudo médico. Analisando detalhadamente as amostras do período que coincide na utilização do método tradicional com a proposta, é possível constatar que médias da pressão arterial e da frequência cardíaca, são bem semelhantes. Desse modo, considerando esse cenário, pode-se confirmar a robustez e a eficácia das ferramentas computacionais utilizadas na proposta.

O cadastramento do voluntário/paciente e anamnese inicial que é realizado no aplicativo móvel e que os dados posteriormente serão utilizados na proposta, como parte dos preditores que serão avaliados no escore de risco de Framingham para hipertensão [PARIKH et al, 2008]. O dashboard carrega as informações cadastradas no aplicativo móvel relacionadas ao voluntário/paciente e nos preditores faltantes necessários para o escore de risco de Framingham é disponibilizado a possibilidade de seleção. No "Laudo" apresentado na Figura 2, o relatório gerado com os dados inseridos e captados, fornecendo a média da medições da pressão arterial e frequência cardíaca; o risco do monitorado ter um episódio cardíaco relacionado a hipertensão nos intervalos de 1, 2 e 3 anos; gráficos relacionados a proposta apresentada, inclusive realizando o descritivo entre os períodos da virgília e do sono com as médias das medições realizadas no período de monitorização. 


\section{DyHEARTMon Risco Individual Geral Detalhamento Especifico Laudo}

O risco do senhor(a) Marcus ser acometido de um incidente relacionado a hipertensão de acordo com a média geral das medições realizadas com o seus preditores é:

1 ano: $3 \%$
2 anos: $6 \%$
3 anos: $13 \%$

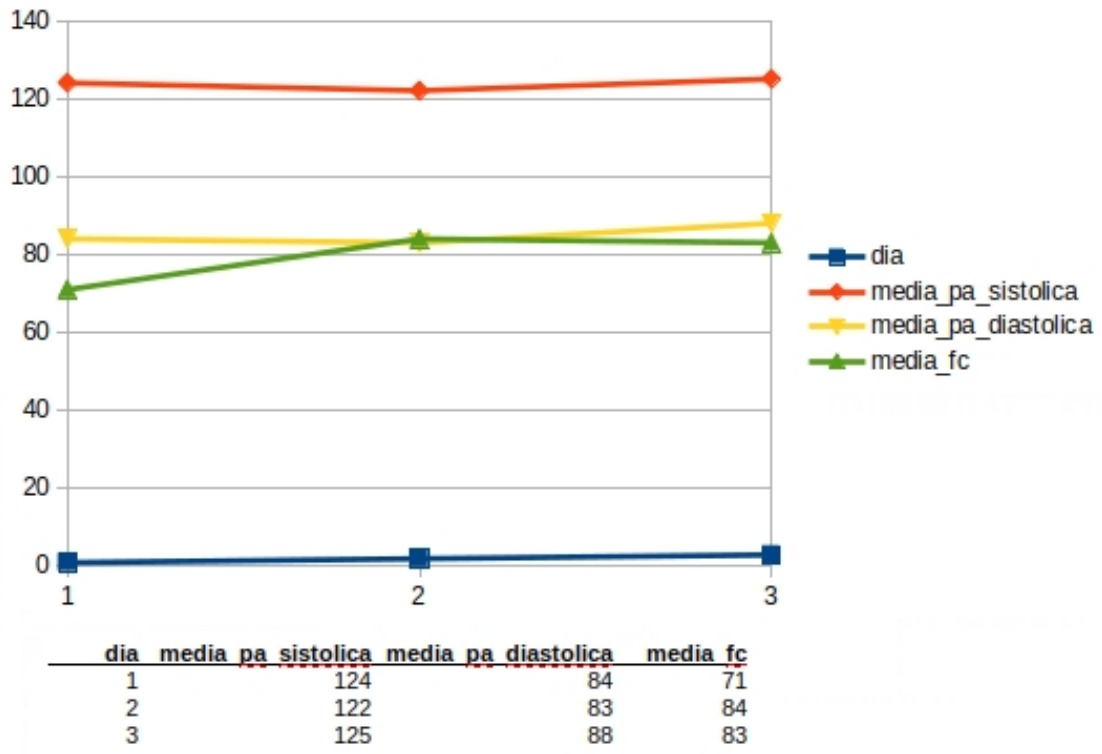

Figura 2. Tela do Laudo

Dois questionários, sendo um aplicado em voluntários e o outro aplicado na entrevista individual com médicos cardiologistas foram utilizados para garantir que as questões relacionadas ao estudo fossem contempladas, além de coletar pontos de melhorias relacionados ao uso da nova proposta de monitorização da pressão arterial.

Para avaliar a usabilidade da arquitetura proposta, foram convidados cinco voluntários que estivessem na faixa etária entre 30 e 65 anos e que pelo uma vez já realizaram o exame convencional da MAPA, para utilizar no período de 72 horas o smartband com o smartphone, realizando todas as atividades diárias, inclusive no período de descanso. Após o período de monitorização, um questionário foi apresentado aos voluntários composto por 5 perguntas objetivas apresentando 4 opções de marcação. Com relação a aceitação da arquitetura, todas as questões que eram esperadas respostas favoráveis a obtiveram. No que se diz a rejeição do paciente ou comprometimento na realização das atividades habituais, o quantitativo de respostas apresentou ser muito pouco provável. Com relação às respostas subjetivas, os voluntários destacam que a proposta ressalta comodidade e praticidade.

Já para a validação, a proposta foi apresentada de maneira individual, para cinco médicos especialistas em cardiologia. Com relação a aceitação do sistema, todas as questões que eram esperadas respostas favoráveis a tiveram. No que se diz a rejeição do paciente e profissionais da saúde, todos os médicos informaram ser nem um pouco provável apresentar alguma rejeição quanto ao uso. Com relação ao comparativo com o exame tradicional, a questão que era esperada resposta favorável a obteve. No que diz respeito a avaliar o risco de influenciar negativamente a avaliação ou tratamento, o quantitativo de respostas apresentou ser muito pouco provável este risco. Com relação ao aumento do período médio da monitorização, para que fosse avaliado se traz a melhora do processo, a questão que era esperada um resposta provável todos os 
entrevistados informaram ser extremamente provável influenciar positivamente a avaliação do paciente. Com relação às respostas subjetivas, os médicos destacam positivamente a possibilidade do acompanhamento dinâmico e apresentar o potencial risco cardíaco.

\section{Considerações finais}

A arquitetura proposta se mostrou bastante promissora pela sua praticidade e comodidade com relação a utilização, dando ênfase a menor possibilidade do efeito do avental branco e melhor mobilidade proporcionada para as atividades diárias do paciente conforme descrito pelos voluntários, inclusive em período superior ao utilizado pelo exame tradicional para captação da pressão arterial e frequência cardíaca. Como uma das principais contribuições do trabalho, tem-se que foi possível associar o uso da internet das coisas como ferramenta computacional para uma proposta alternativa na realização de exames tradicionais para o diagnóstico de doenças cardiovasculares, agregando a possibilidade de recursos de monitoramento online e analise preditiva disponibilizou uma gama de oportunidades para um melhor e mais rápido diagnóstico.

\section{Referências}

BERWANGER, O. (2005). Estratificação de risco cardiovascular: necessitamos de novos marcadores para identificação do paciente de alto risco? Revista da Sociedade de Cardiologia do Rio Grande do Sul, n.6, p. 4-7.

DAVIGNON, J.; GANZ, P. (2004). Atherosclerosis: evolving vascular biology and clinical implications. Role of endothelial dysfunction in atherosclerosis. Circulation, v. 109, suppl. III, p. III-27-III-32.

PARIKH, N. I. et al. (2008). A risk score for predicting near-term incidence of hypertension: The Framingham Heart Study. Annals of Internal Medicine, v. 148, n. 2, p. 102-110., Doi: 10.7326/0003-4819-148-2-200801150-00005.

VIGITEL - Vigilância de Fatores de Risco e Proteção para Doenças Crônicas por Inquérito Telefônico. Ministério da Saúde, (2018). http://portalms.saude.gov.br/vigilancia-em-saude/indica-dores-de-saude/vigilanciade-fatores-de-risco-e-protecao-para-doencas-cronicas-por-inquerito-telefonico-vigitel. 